\title{
Animal models of chronic wound care: the application of biofilms in clinical research
}

This article was published in the following Dove Press journal:

Chronic Wound Care Management and Research

I November 2016

Number of times this article has been viewed

\author{
Hannah Trøstrup' \\ Kim Thomsen' \\ Henrik Calum ${ }^{2}$ \\ Niels Høiby ${ }^{1,3}$ \\ Claus Moser' \\ 'Department of Clinical Microbiology, \\ Copenhagen University Hospital, \\ Rigshospitalet, ${ }^{2}$ Department of \\ Clinical Microbiology, Copenhagen \\ University Hospital, Hvidovre, \\ ${ }^{3}$ Institute for Immunology and \\ Microbiology, University of \\ Copenhagen, Copenhagen, Denmark
}

Correspondence: Hannah Trøstrup Department of Clinical Microbiology, 930I, Copenhagen University Hospital, Rigshospitalet, Juliane Maries vej 22, 2100 DK, Denmark

Tel +4535453545

Fax +4535456412

Email htroestrup@gmail.com

\begin{abstract}
Chronic wounds are a substantial clinical problem affecting millions of people worldwide. Pathophysiologically, chronic wounds are stuck in the inflammatory state of healing. The role of bacterial biofilms in suppression and perturbation of host response could be an explanation for this observation. An inhibiting effect of bacterial biofilms on wound healing is gaining significant clinical attention over the last few years. There is still a paucity of suitable animal models to recapitulate human chronic wounds. The etiology of the wound (venous insufficiency, ischemia, diabetes, pressure) has to be taken into consideration as underlying pathophysiological mechanisms and comorbidities display tremendous variation in humans. Confounders such as infection, smoking, chronological age, sex, medication, metabolic disturbances, and renal impairment add to the difficulty in gaining systematic and comparable studies on nonhealing wounds. Relevant hypotheses based on clinical or in vitro observations can be tested in representative animal models, which provide crucial tools to uncover the pathophysiology of cutaneous skin repair in infectious environments. Disposing factors, species of the infectious agent(s), and time of establishment of the infection are well defined in suitable animal models. In addition, several endpoints can be involved for evaluation. Animals do not display chronic wounds in the way that humans do. However, in many cases, animal models can mirror the pathological conditions observed in humans, although discrepancies between human and animal wound repair are obvious. The use of animal models should be refined and replaced whenever possible, and reproducibility and clinical relevance should be strived. This review aimed at giving an overview of the model systems and major findings for inspiration for clinicians and researchers involved in handling chronic nonhealing wounds. Relevant animal models on wound repair are discussed, and our novel wound model on the host/pathogen interplay is presented. In this model, murine wounds are stuck in a polymorphonuclear neutrophil granulocyte-dominated inflammation due to the presence of visually confirmed Pseudomonas aeruginosa biofilm located in the dermis and subcutaneous fatty tissue.
\end{abstract}

Keywords: pathogen interplay, chronic wound science, Pseudomonas aeruginosa biofilm

\section{Introduction}

The incidence of chronic wounds and its associated complications are growing epidemically, and the management of infections, for example in diabetic foot ulcers, is crucial for the patients. ${ }^{1}$ To date, no known animal model is representative of clinically important comorbidities preceding the formation of a chronic wound.

Pathology of human chronic wounds is a challenge to researchers. In clinical studies, strict inclusion criteria ensure group homogeneity to a certain extent, but 
conclusions are not easily drawn from studies of these often multimorbid patients. In patients suffering from venous insufficiency, accumulation of fibrinous exudates in the lower extremities may form substantial edema. With time, stasis dermatitis and lipodermatosclerosis will develop followed by minor defects in the skin barrier, ultimately causing venous leg ulcers that are difficult to reproduce in animal models due to the complexity of human pathophysiology. However, animal models with venous hypertension that cause altered flow and inflammation are described. ${ }^{2}$ It is believed that diabetic wounds develop due to a reduced healing process as a consequence of impaired cellular functions. ${ }^{1}$ Diabetic wound healing models are well described by Watters et al who have developed a wound model on type 1 diabetic mice. ${ }^{3}$ In this model, biofilm-like aggregates of bacteria in wound sections are visualized. The need for type 2 diabetic models is perhaps even more urgent. Pressure wounds can arise relatively rapidly during continued pressure. In one integrated clinical study, in vivo and in vitro studies were reviewed revealing that pressure ulcers develop in 1 to 4-6 hours. ${ }^{4}$ A porcine model on pressure wounds is suggested by Hyodo et al who applied a constant pressure over denervated skin. ${ }^{5}$ Atherosclerotic disease in extremities is a prerequisite for the development of painful ischemic chronic wounds. Skin flap models reproduce this ischemia. ${ }^{6}$

Despite these clinically relevant models that reflect parts of wound chronicity, there is still an urgent need for animal models that reproduce the inflammatory state that characterizes chronic wounds. An explanation for hyperinflammation could be the presence of bacterial biofilms residing deeply in the wound bed, perturbing normal tissue regeneration.

\section{Choice of animal species}

While choosing a certain species for an animal wound healing model, the comparability of animal skin anatomy to human skin should be taken into consideration. In animal models, a standardized structural damage is inflicted to the skin, whereas in humans, several factors, even some unknown factors, add to the appearance of a recalcitrant wound. Furthermore, human chronic wounds are most often full-thickness wounds, but some animal models utilize only partial-thickness wounding. ${ }^{7}$

A successful animal model on wound repair enables researchers to identify certain mechanisms or key molecules in skin regeneration. A clinically relevant animal model should display impact on wound healing capacity postchallenge. However, a gap exists between the current science on animal wound healing and the development of new clinical treatment modalities. Animal models reflecting chronicity of wounds may only have distinct strengths characterizing different aspects of nonhealing wounds as the reproducibility of more confounding factors are almost impossible to obtain in one animal model. Choice of model system is therefore highly dependent on the hypothesis which has to be tested. Moreover, an already established model may be subject to ongoing adjustments in order to enable careful testing of the generated hypotheses.

The most widely used animals for wound healing models are rodents and larger mammals.

\section{Rodents}

Immune response to infection between species can only be a reflection of clinical matter. Qualitative differences in host response to wounding are a challenge in the use of rodents. ${ }^{8}$ Small rodents such as mice are extensively used as models for skin repair, many offering several advantages. Rat and rabbit physiology is closer to that of humans, but fewer immunological evaluation tools of host response are available. The hairless guinea pig model is closer to human skin anatomy, ${ }^{9}$ which also meets the limitations regarding comparability in host response.

Due to low cost, easy handling, and the fact that rodents are readily obtainable, it enables researchers to include a relatively large number of animals. Intervariability in host response even in inbred strains of mice is inevitable, but is nevertheless reduced as compared to outbred strains. In addition, a substantial number of knockout mouse strains and a vast selection of immunological tools for the evaluation of host response are available for murine models. Genetically modified mice are helpful in the elucidation of the hyperinflammatory state of nonhealing. ${ }^{8}$ However, using small rodents to reflect human wound closure has limitations. For example, human skin has a thicker epidermis ${ }^{10}$ and a more dense appendage density due to hair follicles ${ }^{11}$ than murine skin. Importantly, murine healing is dominated by myofibroblast-mediated contraction due to the presence of striated panniculus carnosus, which causes healing by primary intention and not secondary intention, which is observed in humans. ${ }^{12}$ One method to overcome wound contraction is by the use of a rabbit ear model $^{13,14}$ in which the underlying cartilage functions as a splint, thereby minimizing wound contraction. This model is commonly used for the study of topical growth factors and for the assessment of Pseudomonas aeruginosa (PA) biofilm in wounds. ${ }^{15,16}$ Another way of bypassing wound contraction is by the actual splinting of murine wounds, for 
example, described in Schierle et $\mathrm{al}^{17}$ and Galiano et $\mathrm{a} \mathrm{l}^{14}$ in which wounds heal by the formation of granulation tissue. In these studies, the questionable translatability of acutely inflicted wound to clinically chronic wounds should be addressed and the presence of local wound biofilm should however be visualized.

\section{Larger mammals}

Larger animals such as dogs, pigs, ${ }^{18,19}$ and even primates have more human-like physiology of the skin in certain areas; however, these animals are significantly more expensive, not to mention the logistic challenges in the housing of these larger mammals. Fewer tools are available for host response evaluations as compared to rodent tools. In order to choose larger mammal animal models, researchers therefore have to justify the higher costs and fewer possibilities of the evaluation, and this may be the explanation for the sparse literature on for example, porcine wound healing despite the greater comparability to human skin. ${ }^{18}$ An advantage in porcine wound healing models is that the skin is devoid of fur but coarse singular hairs and besides their skin is firmly attached to the underlying structures like in humans. ${ }^{10}$ However, porcine skin is less vascular than human skin, and it has apocrine, not eccrine, sweat glands. ${ }^{20}$ Summerfield et al have described the similarities between porcine and human immune cells. ${ }^{21}$ Taken together, the impact of these findings on wound healing is not known. ${ }^{18}$

\section{Induction of wounds}

Almost all animal models of chronic wounds are established by the induction of a structural damage of the skin barrier, each model yielding its own defined depth and area. There are numerous methods for generating the skin defect; they include acute surgical wounds, ${ }^{22,23}$ abrasions, punch wounds, extrinsic pressure, ${ }^{24}$ scalding, or burn wounds. ${ }^{25}$ Acute wounds subjected to a single clinical relevant circumstance such as ischemia or diabetes are therefore the cornerstone in animal models of wound chronicity. ${ }^{26}$

\section{Establishment of a chronic wound environment}

Human skin is constitutively an excellent effective barrier, protecting us from numerous microbial exposures every day. Structural damage of the skin barrier, no matter the nature of the underlying pathology, provides the possibility for microbes to colonize deeper layers of the human skin. ${ }^{27}$ If the fundamental disposing factor is not corrected, the colonization will proceed to the establishment of a permanent state of biofilm infection ultimately perturbing and impairing the host response. ${ }^{28-30}$

Microbes gaining access to exposed subcutaneous fatty tissue will be able to cause local infection as the mature biofilm is resistant to host response and antimicrobial therapy. ${ }^{29}$ Human comorbidity such as systemic disease with chronically elevated inflammatory markers, malnutrition, fluctuating glucose levels, or neuropathies is a conducive microenvironment for a biofilm establishment.

In a moist hypoxic and nutritious environment, bacteria will thrive and the net result is a prolonged or chronic inflammatory state delaying or outright impairing wound healing. ${ }^{27,28}$

Biofilm inhibits innate host response ${ }^{29,31}$ and remains refractory to relevant antibiotics. Cascades of collateral damage locally in the tissue decreases the possibility for the wound to heal. ${ }^{32}$ The actual mechanisms causing the pathology around biofilms are not completely understood, but there is a linkage to protease activity and immunosuppression. ${ }^{31,33}$ A suitable animal model should display this prolonged inflammatory state, characterized by constant release of cytokines causing local tissue inflammation. Furthermore, biofilm-like structures should be able to be visualized deeply in the wound bed.

\section{Bacterial biofilms in chronic wound science}

Cutaneous wound healing is a well-orchestrated and dynamic process (Figure 1) that runs through three phases: inflammation, tissue proliferation, and tissue remodeling. ${ }^{34}$ Human chronic wounds are characterized by senescent fibroblasts, a hyperproliferative and thickened epidermis, and prolonged release of proinflammatory cytokines, arresting the wound bed in a chronic inflammatory state. A reason for this arrest is essential in the understanding of recalcitrance of wounds.

The role of bacterial biofilms in chronicity of wounds is only recently appreciated. ${ }^{7}$ James et al found biofilms in 30 of 50 chronic wounds, but only in one in 16 acute wounds, ${ }^{32}$ which is in accordance with the general belief that approximately half of all chronic wounds contain biofilm. ${ }^{35}$ Common bacteria found in chronic wounds are Staphylococcus aureus (SA), Enterococcus species, PA, Proteus species, and anerobic bacteria ${ }^{36,37}$ with some discrepancies observed between wounds of different etiology. ${ }^{38}$ An infectious burden of $>10^{5}$ microbes/g tissue is traditionally considered as infection; ${ }^{39}$ however, classical clinical signs of acute inflammation (calor, dolor, rubor, and tumor) are not always present in diabetic patients ${ }^{40}$ or in chronic low-grade biofilm infections. ${ }^{41} \mathrm{~A}$ clear 


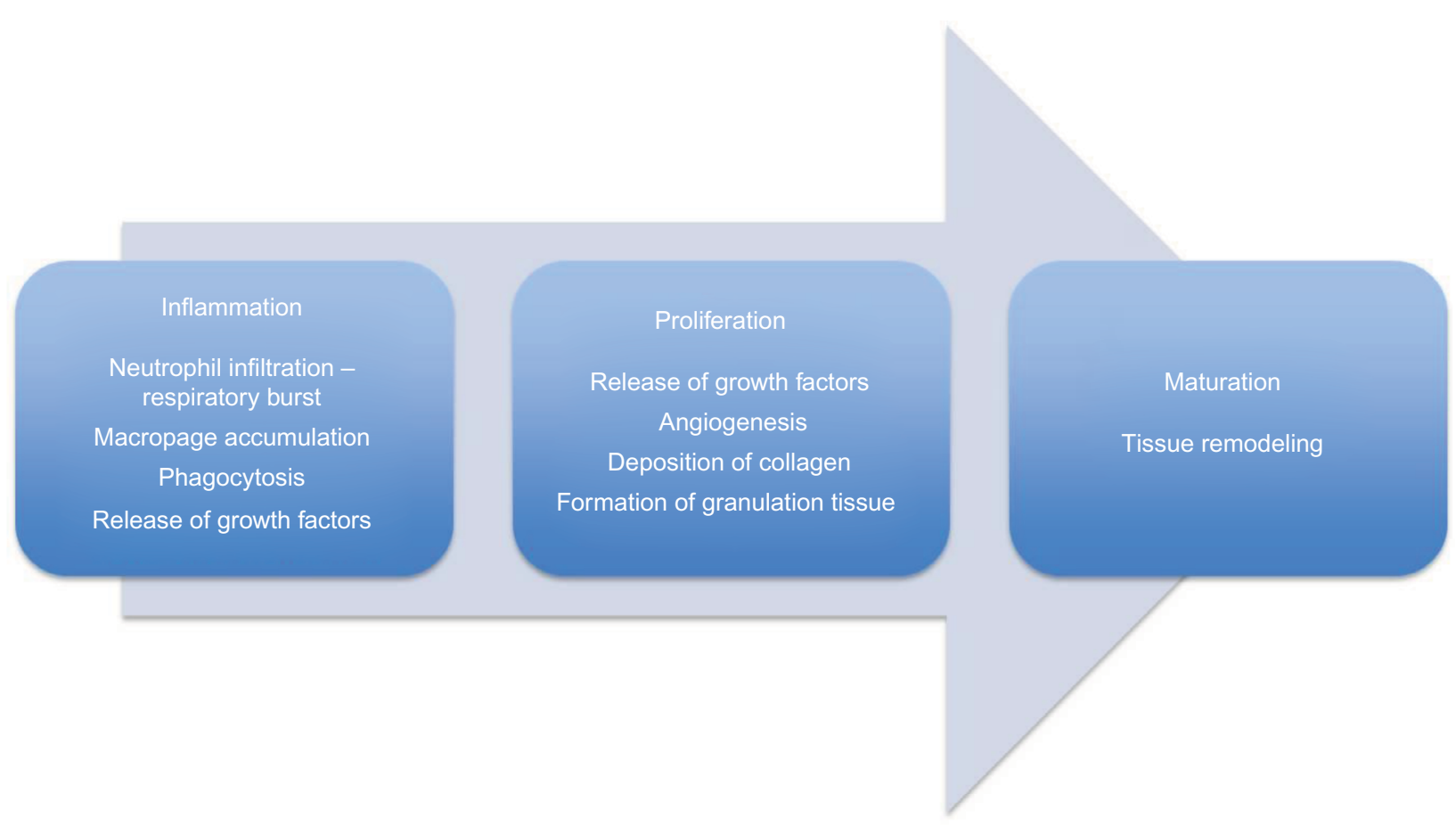

Figure I Normal wound healing is characterized by three dynamic and timely overlapping phases: inflammation, proliferation, and maturation.

Note: The chronic wounds are believed to be stuck in the inflammatory phase of wound healing, which in a healing environment will last for only I-2 days.

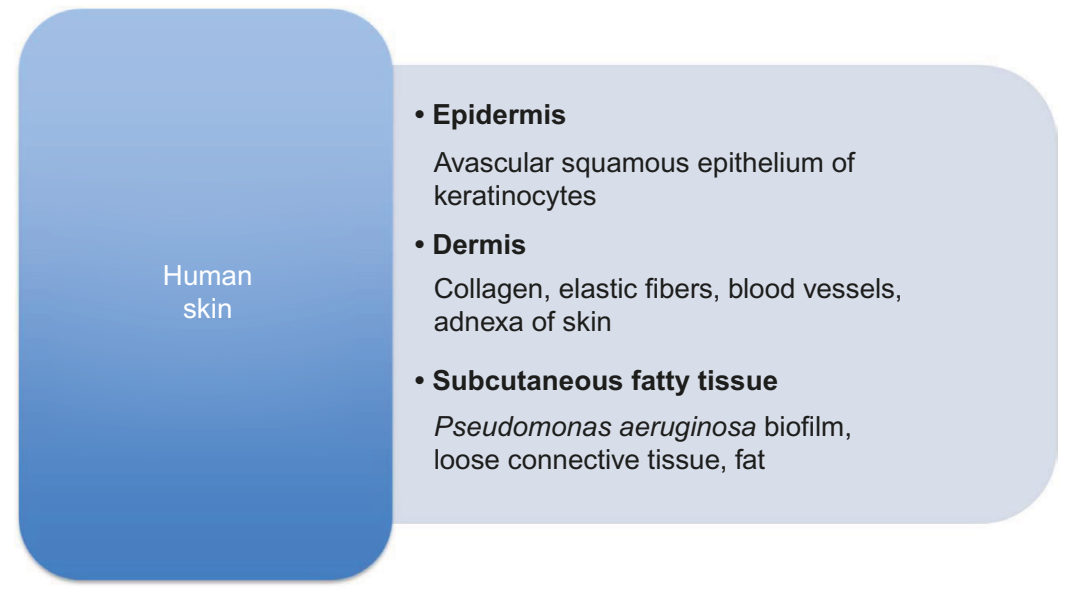

Figure $2 \mathrm{~A}$ diagram of human skin anatomy and contents of the anatomical layers. Note: Note the subcutaneous localization of Pseudomonas aeruginosa biofilm.

distinction between bacterial colonization and clinical infection would be of tremendous value. ${ }^{42}$

The microbiology of chronic wounds of different origins has been somewhat of a black box for many years for several reasons. The sampling of wounds with no classical signs of infection is neglected, and furthermore, Gram-negative rods are often not identified as specimen and no susceptibility pattern is retrieved as this flora is considered nonpathogenic resident bacteria of the chronic wound.$^{28}$ However, PA growing in biofilms is now appreciated as a key role player in arresting wounds in the inflammatory state of healing. ${ }^{35,43} \mathrm{An}$ uneven distribution of microorganisms has been described. PA biofilms are located in the subcutaneous fatty tissues of the wounds ${ }^{44}$ (Figure 2). Comparing different species, PA tends to be located deeper in the wound bed than SA, ${ }^{44,45}$ and in tissue biopsies from chronic wounds, the former appeared more virulent than the latter, evaluated by the number of neutrophils accumulating at the site. ${ }^{46}$ Thus sampling technique is of utmost importance - especially, biopsies from surgical revision have proven to be superior in revealing the actual microbiology of the wounds as compared to conventional swabbing. ${ }^{35,43}$ 
Table I Some examples of animal models on chronic wounds

\begin{tabular}{|c|c|c|c|c|}
\hline Animal species & Wound etiology & Microbe & Duration of experiment & Authors \\
\hline Cyclophosphamide-treated mice & Cut wound & $3.7 \times 10^{6}$ Staphylococcus aureus & 60 hours & Akiyama et $\mathrm{al}^{22}$ \\
\hline Pigs & $\begin{array}{l}\text { Partial thickness by the use of } \\
\text { electrokeratome }\end{array}$ & $\begin{array}{l}10^{7} \mathrm{CFU} / \mathrm{mL} \\
\text { Staphylococcus aureus }\end{array}$ & 48 hours & Davis et $\mathrm{al}^{70}$ \\
\hline Mice & Full-thickness punch wound & $\begin{array}{l}\text { Staphylococcus aureus } \\
\text { Staphylococcus epidermidis }\end{array}$ & 9 days & Schierle et al $^{17}$ \\
\hline Diabetic mice & Full-thickness excision wound & $10^{4}$ Pseudomonas aeruginosa & 16 days & Watters et al ${ }^{62}$ \\
\hline Diabetic mice & Full-thickness punch wound & Pseudomonas aeruginosa & 28 days & Zhao et $\mathrm{al}^{49}$ \\
\hline Rabbits & Full-thickness punch wound & $10^{6}$ Staphylococcus aureus & 12 days & Gurjala et al ${ }^{16}$ \\
\hline Cyclophosphamide-treated mice & Full-thickness punch wound & $5 \times 10^{4}$ Acinetobacter baumanii & 23 days & Thompson et $\mathrm{al}^{71}$ \\
\hline
\end{tabular}

In one study, PA was significantly more often identified in wound material as compared to swabs. ${ }^{35}$ Another clinical relevance of PA is that infection by this microbe reduces the success rate in split-thickness grafting of chronic venous leg ulcers. ${ }^{47}$ It may also contribute to the large area of leg ulcers. ${ }^{36}$ Wound biopsies or other wound tissue can be examined by light microscopy after Gram-staining or by fluorescence microscopy after staining with fluorescent in situ hybridization (FISH) techniques - especially the use of peptide nucleotide acid (PNA) probes has proven useful in revealing biofilms residing within the nonhealing wounds. ${ }^{35}$

Animal models of chronic wounds caused by biofilms are emerging, but this research area is still not thoroughly investigated. The idea is that the biofilm in itself creates a chronic infection, and one of the biggest challenges in the creation of a representative animal model is to somehow mimic this low-grade inflammation. Due to the described observations, the recent animal models have focused on embedding bacteria inside biofilms or induce biofilm growth and organization before establishing the wound infection in the animals. ${ }^{48}$ Examples of relevant animal models on chronic wounds are summarized in Table 1.

In a dermal punch wound model on rabbit ears, SA biofilm creates a low-grade and persistent host response, impairing epithelialization and granulation tissue formation. ${ }^{16}$ Zhao et al developed a reproducible full-thickness diabetic punch wound model on mice challenged by in vitro produced PA biofilm. ${ }^{49}$ They reported a PA biofilm-induced impairment of wound healing. In this model, bacteria in biofilms are located in the scab of wounds, which is not the case in humans. ${ }^{49,50}$ In another full-thickness excisional wound murine model, Dalton et al demonstrated impaired wound healing in a polymicrobial biofilm infection model in comparison with single species infection, suggesting a significant contribution of interspecies interactions on delayed healing ${ }^{48}$ Interestingly, an antimicrobial dressing was found counteracting the detrimental PA biofilm effects on wound healing underlining the usefulness of such models to perform preclinical testing of potential beneficial treatment strategies. ${ }^{51}$

Recalcitrance to antibiotics of biofilms ${ }^{52-55}$ can to a high degree explain why antibiotics are not convincingly effective in handling nonhealing wounds. Necessary antibiofilm strategies of high doses and combination therapy combined with suitable debridement are suggested.

\section{Endpoints for wound healing assessment}

Clinically relevant endpoints as a parameter for the general condition of the animal are daily weighing and temperature assessment. Various clinical scoring systems are available - besides giving the opportunity to obtain repeated measurements on the same animal; this is actually a mandatory evaluation of the status of any animal used for experiments..$^{56}$

In human studies, the primary end point is the appearance of granulation tissue, the reduction of wound area or ultimately reepithelialization. In case of evaluation of efficacy of a given treatment, the type of sample and technique used to obtain the material are of substantial significance. Biofilms cannot be identified and reported from a swab due to the superficial nature of the sample. ${ }^{35}$ Another issue is that the microbiological examinations are not performed uniformly or standardized, and often not everything cultured is identified or even reported as clinicians often only retrieve the material when the wound appears clinically infected with the classical signs of inflammation. With the development of newer molecular techniques, genetic material from numerous different pathogens can be detected in wound biopsies or discarded material from revisions..$^{57}$ So-called microbiomes of the wounds can vary over time. ${ }^{58}$ However, the actual clinical significance of these findings by the next generation molecular techniques still needs to be solved. Interestingly, a recent case study demonstrated the dynamics of bacterial constituents during the course of wound healing. Initially Enterobacteriaceae and SA dominate the flora, 
and their disappearance during antibiotic treatment seems to initiate wound healing even though the bacterial load remains stationary. Thus, the control of certain pathogens seems to favor a healing process while the presence of other microorganisms is indifferent. ${ }^{59}$

Wound closure is perhaps the most important clinical parameter in following patients with nonhealing wounds. ${ }^{60}$ Laboratory animals can be followed in the same manner by digital planimetry, for example, in the assessment of murine skin contraction. ${ }^{61}$ Photos of the wounds are retrieved in a standardized manner (distance from wound and precisely over the middle of the wound) and are subsequently transferred to a computer-based program for estimating the sizes of the wounds throughout the assessment period. These can then be compared with the initial sizes of the affected areas, expressing healing as percentage of wound closure by the equation $\left(A_{0}-A_{\mathrm{t}}\right) / A_{0} \times 100,{ }^{60,62}$ in which $A_{0}$ is the area of the wound at the beginning of the experiment, and $A_{\mathrm{t}}$ is the present wound area. Such clinical assessment with repeated measurements on the same animals has the potential to provide strong statistical analysis with paired parameters since each animal functions as its own control.

In vivo imaging systems using fluorescent or bioluminiscent microorganisms can be useful for the assessment of wound infections in animal models. They provide repeated measurements on the same animals, following the course of the infections, thus reducing the number of experimental animals required. The superficial localization of infection in the wound enables exciting new perspectives by means of in vivo imaging systems in chronic wound science. ${ }^{63}$

\section{An example of a chronic PA biofilm infection model}

Biofilms induce a persistent cellular inflammatory response evaluated by the accumulation of polymorphonuclear neutrophil (PMN) granulocytes and elevated levels of interleukin- $1 \beta .{ }^{64}$ Our chronic wound model is a modification of our murine burn wound model. ${ }^{65} \mathrm{~A}$ thermal lesion provides the structural damage, a full-thickness necrosis, necessary for the establishment of a chronic biofilm infection. ${ }^{66}$ This model provides the possibility to study the impact of PA biofilms on wounded skin in vivo. A third-degree burn to anesthetized mice was induced, the burn taking up $6 \%$ of the total body surface area (Figure 3). To circumvent systemic immunosuppression caused by the burn, PA biofilm was injected 4 days after wound infliction. In order to mimic the spatial distribution of bacteria observed clinically, the challenge solution is injected deep in the wound bed to reach the dermis and the subcutaneous fatty layer (Figure 2). If planktonic PA is injected subcutaneously beneath the affected skin of mice, systemic dissemination and spreading to the organs yielding severe sepsis 2 days post challenge is observed. ${ }^{66}$ However, if the bacteria are embedded in seaweed alginate to mimic biofilm organization, no systemic spread of bacteria is observed and the mice continue to be locally infected for a minimum of 14 days. ${ }^{65}$

Using specific PA staining with PNA-FISH on in toto removed wounds from these mice, biofilm-like structures in both dermis and subcutaneous layers of the skin were revealed. ${ }^{65}$ Inflammatory cells were accumulated in close proximity to the biofilms. As for quantitative bacteriology, a significantly larger amount of bacteria detected by counting of $\mathrm{CFU} /$ wound in BALB/c mice as compared to $\mathrm{C} 3 \mathrm{H} / \mathrm{HeN}$ was found at day 5 . When looking at the course of infection, $\mathrm{BALB} / \mathrm{c}$ mice had significantly more bacteria at day 5 than day 2 , and a significant reduction again at day 7 as opposed to $\mathrm{C} 3 \mathrm{H} / \mathrm{HeN}$. In this inbred mouse strain, quantitative bacteriology was stable throughout the study.

A significant observation was the arrested inflammation in a state dominated by PMNs in mice with established biofilm infection using this model. This was in contrast to the normal shift of inflammation to a cellular response with mononuclear cells observed in mice challenged with alginate beads without PA. Furthermore, levels of interleukin- $1 \beta$ are elevated locally in the biofilm-infected wounds, especially in the relatively susceptible BALB/c-strain of mice, which are immunologically characterized by a Th2-dominating response. ${ }^{65}$ This strain did not reach infection control during the experiment as opposed to $\mathrm{CH} 3 / \mathrm{HeN}$ mice. ${ }^{65}$ Interestingly, chemoattractant for PMNs, keratinocyte-derived chemokine, was significantly higher at both day 2 postinfection and day 5 in serum from PA-infected BALB/c mice but only day 2 postinfection for the $\mathrm{C} 3 \mathrm{H} / \mathrm{HeN}$ strain.

This novel animal model mimics human nonhealing wounds, arrested in a hyperinflammatory state evaluated by PMN infiltration and interleukin-1 $\beta$. The arrest is caused by the presence of biofilm-like structures situated deeply in the murine wound beds.

Quantitative bacteriology analysis on homogenized total excised wounds was always included as an endpoint, excluding animals with wounds colonized with additional microorganisms acquired after the initiation of the experiments. In addition, microscopy of excised wounds by use of PNA-FISH techniques is occasionally included to show the presence of biofilms within the wounds. ${ }^{65}$ 
A

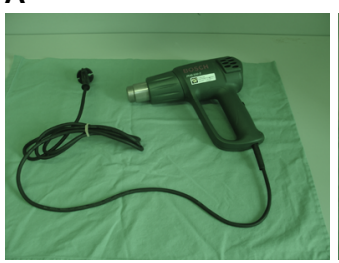

E

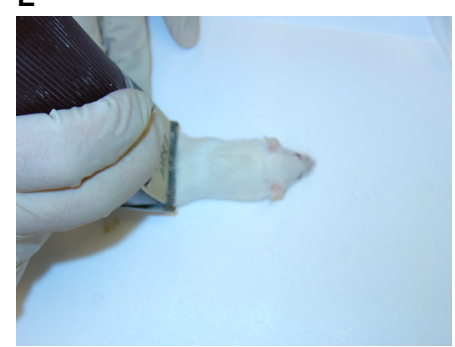

$\mathbf{F}$
C

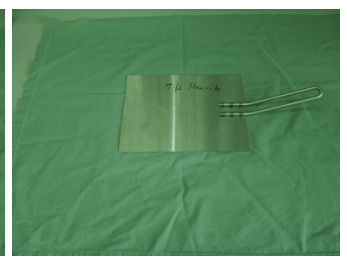

G
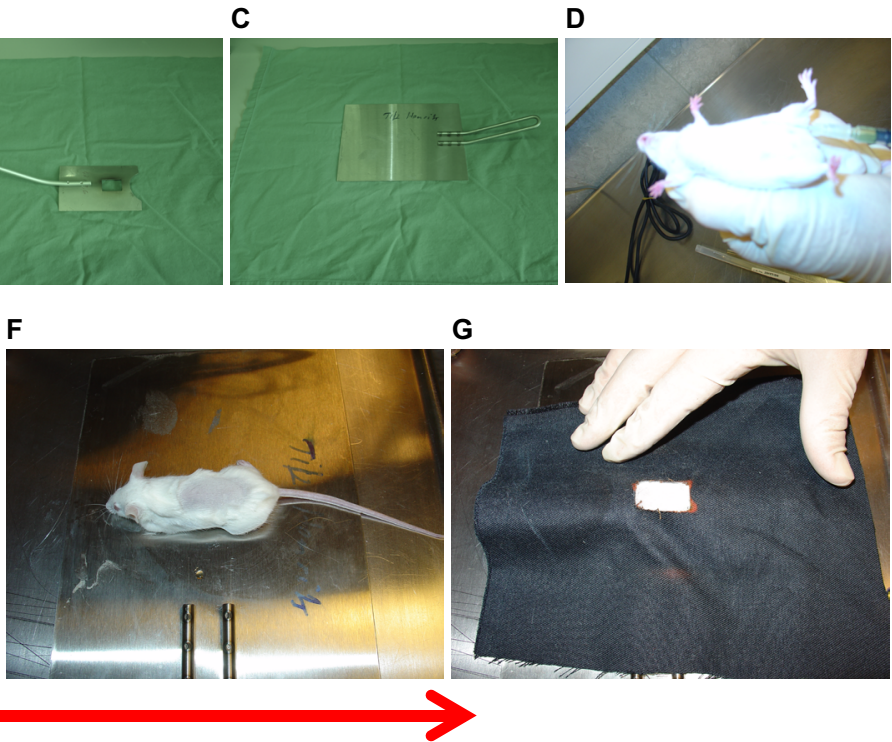

H

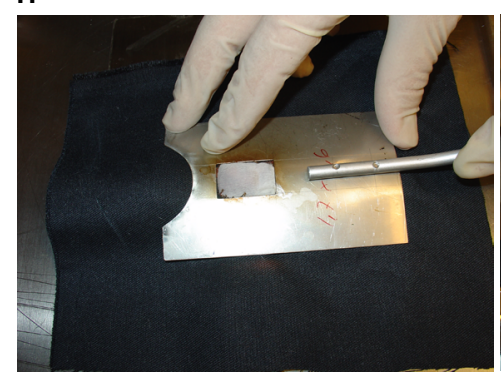

I
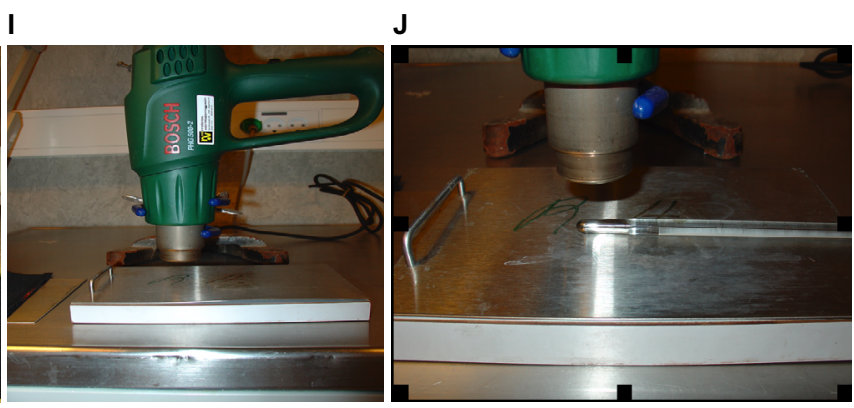

K

L

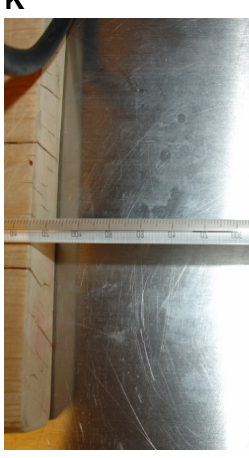

$\mathbf{N}$

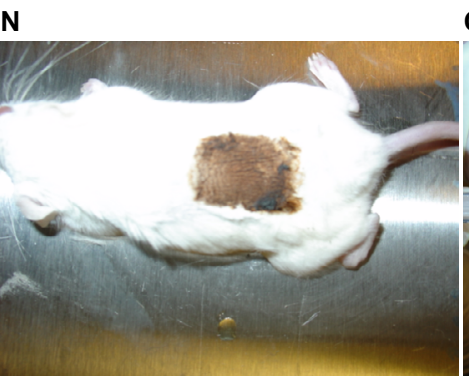

o

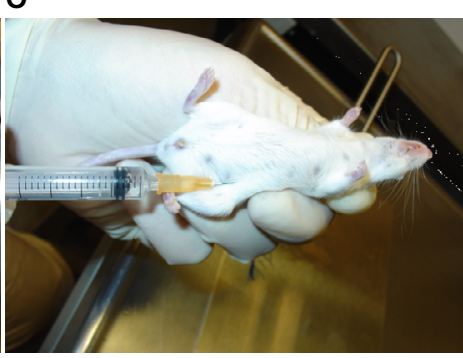

M

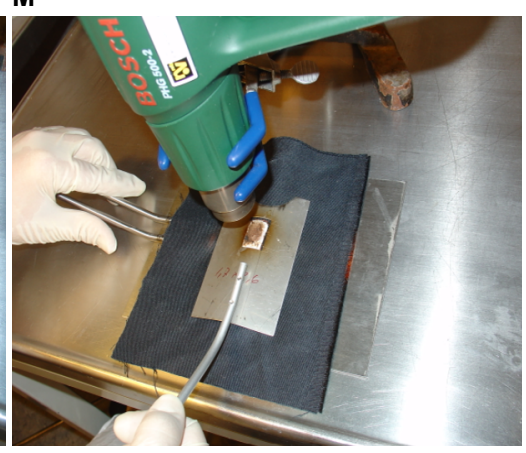

$\mathbf{P}$

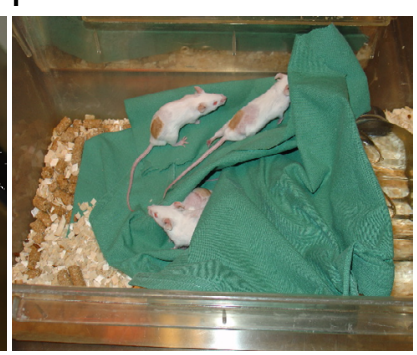

Figure 3 The procedure.

Notes: (A) The hot air blower, (B) the template with window, $(\mathbf{C})$ the sledge, $(\mathbf{D})$ injection of hypnorm dormicum SC, (E) shaving the mouse, (F) placing the mouse on the sledge, $(\mathbf{G})$ covering the mouse with fire blanket, $(\mathbf{H})$ placing the template on the mouse, $(\mathbf{I})$ the arrangement, $(\mathbf{J})$ measurement of the temperature, $(\mathbf{K}) 330^{\circ} \mathrm{C},(\mathbf{L})$ initiating the induction of the thermal injury, $(\mathbf{M})$ keeping the mouse for $7 \mathrm{~s}$ into the hot air, ( $\mathbf{N})$ clinical manifestation of the thermal injury, $(\mathbf{O})$ injection of $\mathrm{I}$ ml of $\mathrm{NaCl}$, $(\mathbf{P})$ the mice 4-5 h after induction of the thermal injury. Reproduced from Pseudomonas Methods and Protocols, volume II 49 of the series Methods in Molecular Biology. 'Burn Mouse Models', pP 793-802, 29 March 2014. Calum H, Høiby N, Moser C. With permission of Springer. ${ }^{72}$

Abbreviation: SC, subcutaneously. 
A

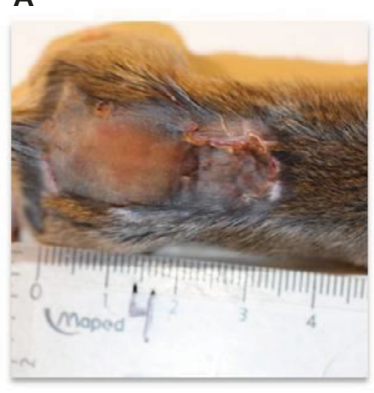

B

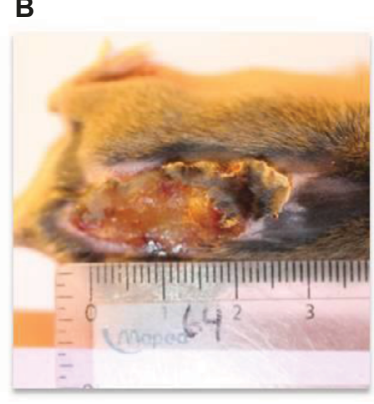

C

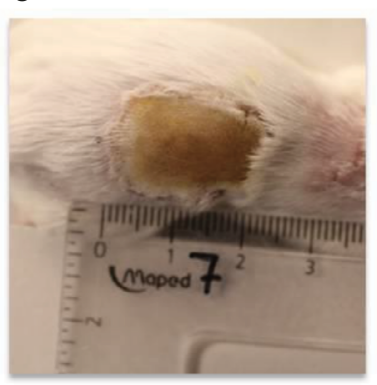

D

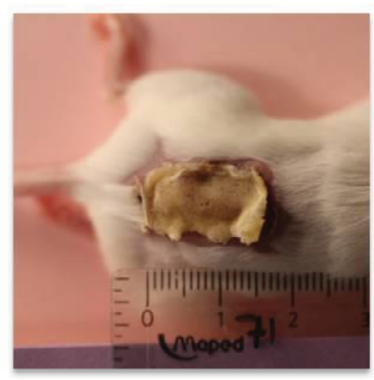

Figure 4 Macroscopic skin contraction in two inbred strains of mice. Notes: Pseudomonas aeruginosa biofilm was injected subcutaneously 4 days after wound infliction in order to bypass systemic immunosuppression caused by the burn procedure. Depicted are the appearance of an infected $\mathrm{C} 3 \mathrm{H} / \mathrm{HeN}$ mouse wound at day 4 (A) and day 10 (B) postinfection. (C) and (D) show infected BALB/c mouse wound at day 4 and day 10, respectively. Digital planimetry can be used in the assessment of wound healing.

Patients suffering from chronic wounds often display comorbidity and therefore have significant increase in systemic inflammatory markers such as leucocytes, C-reactive protein or procalcitonine, or sign of systemic infections. In our model, flow cytometry analysis on whole blood shows significant differences on leucocytes between chronically infected mice and mice without chronic wounds, indicating a systemic impact of the wound infliction. ${ }^{65}$

Numerous inflammatory markers can be revealed locally in the wounds compared with controls in wound exudates collected in a standardized manner ${ }^{67}$ or wound tissue. Unfortunately, no marker has been proven to be a characteristic of nonhealing wounds probably due to the heterogeneity of wound pathology. Animal models have a substantial advantage as the wound removed in toto can be examined after homogenization and the supernatants can be subsequently analyzed by enzyme-linked immunosorbent assay (ELISA), or multiplex fluorescent Luminex immunoassay, or quantitative polymerase chain reaction techniques. ${ }^{65}$

Inflammatory characterization and degree are evaluated histopathologically on excised whole chronic wounds after staining with hematoxylin and eosin. ${ }^{65}$ In a time course of 14 days post wound infliction, BALB/c mice display a larger number of PMNs peripherally in the wounds than centrally

(authors' observations, 2014). Moreover, an arrestment in the proinflammatory stage dominated by PMNs was observed. ${ }^{65}$

This model is an easily obtainable and reproducible method to study the impact of biofilm infection on host response. Furthermore, it can be used for novel experimental treatments such as growth factors in combination with antibiotics or other antibiofilm interventions such as quorum sensing inhibitors, azithromycin, and acetic acid. ${ }^{68,69}$ As a model for wound healing, assessment of wound closure is a possibility (Figure 4). Figure 4 unpublished study was approved by the Animal Ethics Committee of Denmark (2010/561-1766). The authors confirm that all experiments were performed following National and European Union guidelines.

\section{Conclusion}

There is an urgent need for the focus on the host responsebiofilm interaction in wound science, but how to create an in vivo model that recapitulates the complexity of human wound chronicity in an acceptable manner still remains unanswered. Although they are merely approximations of clinical conditions due to phenotypic differences in skin anatomy and host response profile between species, animal models provide an indispensable insight into skin repair.

Current refined models serve as a crucial basis for testing hypotheses observed clinically and in vitro. The ultimate goal using animal models is to identify the key role-players in recalcitrant wound healing. This could be used in the evaluation of treatment options that are clinically beneficial for the growing population of patients suffering from chronic wounds.

\section{Disclosure}

The authors report no conflicts of interest in this work.

\section{References}

1. Jeffcoate WJ, Harding KG. Diabetic foot ulcers. Lancet. 2003;361: 1545-1551.

2. Bergan JJ, Pascarella L, Schmid-Schonbein GW. Pathogenesis of primary chronic venous disease: insights from animal models of venous hypertension. J Vasc Surg. 2008;47:183-192.

3. Watters C, DeLeon K, Trivedi U, et al. Pseudomonas aeruginosa biofilms perturb wound resolution and antibiotic tolerance in diabetic mice. Med Microbiol Immunol. 2013;202:131-141.

4. Gefen A. How much time does it take to get a pressure ulcer? Integrated evidence from human, animal, and in vitro studies. Ostomy Wound Manage. 2008;54:26-28,30-35.

5. Hyodo A, Reger SI, Negami S, Kambic H, Reyes E, Browne EZ. Evaluation of a pressure sore model using monoplegic pigs. Plast Reconstr Surg. 1995;96:421-428.

6. Chen C, Schultz GS, Bloch M, Edwards PD, Tebes S, Mast BA. Molecular and mechanistic validation of delayed healing rat wounds as a model for human chronic wounds. Wound Repair Regen. 1999;7:486-494.

7. Seth AK, Geringer MR, Hong SJ, Leung KP, Mustoe TA, Galiano RD. In vivo modeling of biofilm-infected wounds: a review. J Surg Res. 2012;178:330-338. 
8. Eming SA, Krieg T, Davidson JM. Inflammation in wound repair: molecular and cellular mechanisms. J Invest Dermatol. 2007;127:514-525.

9. Sueki H, Gammal C, Kudoh K, Kligman AM. Hairless guinea pig skin: anatomical basis for studies of cutaneous biology. Eur J Dermatol. 2000;10:357-364.

10. Davidson JM. Animal models for wound repair. Arch Dermatol Res. 1998;290(Suppl):S1-S11.

11. Pasparakis M, Haase I, Nestle FO. Mechanisms regulating skin immunity and inflammation. Nat Rev Immunol. 2014;14:289-301.

12. Davidson JM, Yu F, Opalenik SR. Splinting strategies to overcome confounding wound contraction in experimental animal models. $A d v$ Wound Care. 2013;2:142-148.

13. Ahn ST, Mustoe TA. Effects of ischemia on ulcer wound healing: a new model in the rabbit ear. Ann Plast Surg. 1990;24:17-23.

14. Galiano RD, MichaelsJT, Dobryansky M, Levine JP, Gurtner GC. Quantitative and reproducible murine model of excisional wound healing. Wound Repair Regen. 2004;12:485-492.

15. Seth AK, Geringer MR, Gurjala AN, Hong SJ, Galiano RD, Leung KP, Mustoe TA. Treatment of Pseudomonas aeruginosa biofilm-infected wounds with clinical wound care strategies: a quantitative study using an in vivo rabbit ear model. Plast Reconstr Surg. 2012;129:262e-274e

16. Gurjala AN, Geringer MR, Seth AK, et al. Development of a novel, highly quantitative in vivo model for the study of biofilm-impaired cutaneous wound healing. Wound Repair Regen. 2011;19:400-410.

17. Schierle CF, De la Garza M, Mustoe TA, Galiano RD. Staphylococcal biofilms impair wound healing by delaying reepithelialization in a murine cutaneous wound model. Wound Repair Regen. 2009;17:354-359.

18. Seaton M, Hocking A., Gibran NS. Porcine models of cutaneous wound healing. ILAR J. 2015;56:127-138.

19. Sullivan TP, Eaglstein WH, Davis SC, Mertz P. The pig as a model for human wound healing. Wound Repair Regen. 2001;9:66-76.

20. Montagna W, Yun JS. The skin of the domestic pig. J Invest Dermatol. 1964;42:11-21.

21. Summerfield A, Meurens F, Ricklin ME. The immunology of the porcine skin and its value as a model for human skin. Mol Immunol. 2015;66:14-21.

22. Akiyama H, Kanzaki H, Tada J, Arata J. Staphylococcus aureus infection on cut wounds in the mouse skin: experimental staphylococcal botryomycosis. J Dermatol Sci. 1996;11:234-238.

23. Asada M, Nakagami G, Minematsu T, et al. Novel models for bacterial colonization and infection of full-thickness wounds in rats. Wound Repair Regen. 2012;20:601-610.

24. Nakagami G, Morohoshi T, Ikeda T, et al. Contribution of quorum sensing to the virulence of Pseudomonas aeruginosa in pressure ulcer infection in rats. Wound Repair Regen. 2011;19:214-222.

25. Rashid MH, Rumbaugh K, Passador L, Davies DG, Hamood AN, Iglewski BH, Kornberg A. Polyphosphate kinase is essential for biofilm development, quorum sensing, and virulence of Pseudomonas aeruginosa. Proc Natl Acad Sci U S A. 2000;97:9636-9641.

26. Nunan R, Harding KG, Martin P. Clinical challenges of chronic wounds: searching for an optimal animal model to recapitulate their complexity. Dis Model Mech. 2014;7:1205-1213.

27. Edwards R, Harding KG. Bacteria and wound healing. Curr Opin Infect Dis. 2004;17:91-96.

28. Bowler PG, Duerden BI, Armstrong DG. Wound microbiology and associated approaches to wound management. Clin Microbiol Rev. 2001;14:244-269.

29. Percival SL, McCarty SM, Lipsky B. Biofilms and wounds: an overview of the evidence. Adv Wound Care. 2015;4;373-381.

30. Trøstrup H, Holstein P, Christophersen L, et al. S100A8/A9 is an important host defence mediator in neuropathic foot ulcers in patients with type 2 diabetes mellitus. Arch Dermatol Res. 2016;308:347-355.

31. Jesaitis AJ, Franklin MJ, Berglund D, et al. Compromised host defense on Pseudomonas aeruginosa biofilms: characterization of neutrophil and biofilm interactions. J Immunol. 2003;171:4329-4339.

32. James GA, Swogger E, Wolcott R, et al. Biofilms in chronic wounds. Wound Repair Regen. 2008;16:37-44.
33. Percival SL, Hill KE, Williams DW, Hooper SJ, Thomas DW, Costerton JW. A review of the scientific evidence for biofilms in wounds. Wound Repair Regen. 2012;20:647-657.

34. Singer AJ, Clark RA. Cutaneous wound healing. New Engl J Med. 1999;341:738-746.

35. Kirketerp-Møller K, Jensen PØ, Fazli M, et al. Distribution, organization, and ecology of bacteria in chronic wounds. J Clin Microbiol. 2008;46:2717-2722.

36. Gjødsbøl K, Christensen JJ, Karlsmark T, Jørgensen B, Klein BM, Krogfelt KA. Multiple bacterial species reside in chronic wounds: a longitudinal study. Int Wound J. 2006;3:225-231.

37. Renner R, Sticherling M, Ruger R, Simon J. Persistence of bacteria like Pseudomonas aeruginosa in non-healing venous ulcers. Eur J Dermatol. 2012;22:751-757.

38. McInnes RL, Cullen BM, Hill KE, et al. Contrasting host immunoinflammatory responses to bacterial challenge within venous and diabetic ulcers. Wound Repair Regen. 2014;22:58-69.

39. Robson MC. Burn sepsis. Crit Care Clin. 1988;4:281-298.

40. Cutting KF, White R. Defined and refined: criteria for identifying wound infection revisited. Br J Community Nurs. 2004;9:S6-S15.

41. Høiby N, Bjarnsholt T, Moser C, et al. ESCMID guideline for the diagnosis and treatment of biofilm infections 2014. Clin Microbiol Infect. 2015;21(Suppl 1):S1-S25.

42. Robson MC. Wound infection. A failure of wound healing caused by an imbalance of bacteria. Surg Clin North Am. 1997;77:637-650.

43. Bjarnsholt T, Kirketerp-Møller K, Jensen PØ, et al. Why chronic wounds will not heal: a novel hypothesis. Wound Repair Regen. 2008;16:2-10.

44. Fazli M, Bjarnsholt T, Kirketerp-Møller K, et al. Nonrandom distribution of Pseudomonas aeruginosa and Staphylococcus aureus in chronic wounds. J Clin Microbiol. 2009;47:4084-4089.

45. Burmolle M, Thomsen TR, Fazli M, et al. Biofilms in chronic infections - a matter of opportunity - monospecies biofilms in multispecies infections. FEMS Immunol Med Microbiol. 2010;59:324-336.

46. Fazli M, Bjarnsholt T, Kirketerp-Møller K, Jørgensen A, Andersen CB, Givskov M, Tolker-Nielsen T. Quantitative analysis of the cellular inflammatory response against biofilm bacteria in chronic wounds. Wound Repair Regen. 2011;19:387-391.

47. Høgsberg T, Bjarnsholt T, Thomsen JS, Kirketerp-Møller K. Success rate of split-thickness skin grafting of chronic venous leg ulcers depends on the presence of Pseudomonas aeruginosa: a retrospective study. PloS One. 2011;6:e20492.

48. Dalton T, Dowd SE, Wolcott RD, Sun Y, Watters C, Griswold JA, Rumbaugh KP. An in vivo polymicrobial biofilm wound infection model to study interspecies interactions. PloS One. 2011;6:e27317.

49. Zhao G, Hochwalt PC, Usui ML, et al. Delayed wound healing in diabetic $(\mathrm{db} / \mathrm{db})$ mice with Pseudomonas aeruginosa biofilm challenge: a model for the study of chronic wounds. Wound Repair Regen. 2010;18;467-477.

50. Zhao G, Usui ML, Underwood RA, et al. Time course study of delayed wound healing in a biofilm-challenged diabetic mouse model. Wound Repair Regen. 2012;20:342-352.

51. Seth AK, Zhong A, Nguyen KT, Hong SJ, Leung KP, Galiano, RD, Mustoe TA. Impact of a novel, antimicrobial dressing on in vivo, Pseudomonas aeruginosa wound biofilm: quantitative comparative analysis using a rabbit ear model. Wound Repair Regen. 2014;22:712-719.

52. Moskowitz SM, Foster JM, Emerson J, Burns JL. Clinically feasible biofilm susceptibility assay for isolates of Pseudomonas aeruginosa from patients with cystic fibrosis. J Clin Microbiol. 2004;42:1915-1922.

53. Hengzhuang W, Wu H, Ciofu O, Song Z, Hoiby N. Pharmacokinetics/ pharmacodynamics of colistin and imipenem on mucoid and nonmucoid Pseudomonas aeruginosa biofilms. Antimicrob Agents Chemother. 2011;55:4469-4474.

54. Anwar H, Costerton JW. Enhanced activity of combination of tobramycin and piperacillin for eradication of sessile biofilm cells of Pseudomonas aeruginosa. Antimicrob Agents Chemother. 1990;34: 1666-1671.

55. Cao B, Christophersen L, Thomsen K, et al. Antibiotic penetration and bacterial killing in a Pseudomonas aeruginosa biofilm model. $J$ Antimicrob Chemother. 2015;70:2057-2063. 
56. Thomsen K, Christophersen L, Bjarnsholt T, Jensen PØ, Moser C, Høiby N. Anti-pseudomonas aeruginosa IgY antibodies augment bacterial clearance in a murine pneumonia model. J Cyst Fibros. 2016;15:171-178.

57. Wolcott RD, Hanson JD, Rees EJ, et al. Analysis of the chronic wound microbiota of 2,963 patients by $16 \mathrm{~S}$ rDNA pyrosequencing. Wound Repair Regen. 2016;24:163-174.

58. Grice EA. The skin microbiome: potential for novel diagnostic and therapeutic approaches to cutaneous disease. Semin Cutan Med Surg. 2014;33:98-103.

59. Sprockett DD, Ammons CG, Tuttle MS. Use of $16 \mathrm{~S}$ rRNA sequencing and quantitative PCR to correlate venous leg ulcer bacterial bioburden dynamics with wound expansion, antibiotic therapy, and healing. Wound Repair Regen. 2015;23:765-771.

60. Brown GL, Nanney LB, Griffen J, et al. Enhancement of wound healing by topical treatment with epidermal growth factor. $N$ Engl J Med. 1989;321:76-79.

61. Brown RL, Greenhalgh DG. Mouse models to study wound closure and topical treatment of infected wounds in healing-impaired and normal healing hosts. Wound Repair Regen. 1997;5:198-204.

62. Watters C, DeLeon K, Trivedi, U, et al. Pseudomonas aeruginosa biofilms perturb wound resolution and antibiotic tolerance in diabetic mice. Med Microbiol Immunol. 2013;202:131-141.

63. Kuo YR, Wang CT, Cheng JT, Kao GS, Chiang YC, Wang CJ. Adiposederived stem cells accelerate diabetic wound healing through the induction of autocrine and paracrine effects. Cell Transplant. 2016;25: 71-81.
64. Wolcott RD, Rhoads DD, Bennett ME, Wolcott BM, Gogokhia L, Costerton JW, Dowd SE. Chronic wounds and the medical biofilm paradigm. J Wound Care. 2010;19:45-46,48-50,52-43.

65. Trøstrup H, Thomsen K, Christophersen LJ, et al. Pseudomonas aeruginosa biofilm aggravates skin inflammatory response in BALB/c mice in a novel chronic wound model. Wound Repair Regen. 2013;21:292-299.

66. Calum H, Moser C, Jensen PØ, et al. Thermal injury induces impaired function in polymorphonuclear neutrophil granulocytes and reduced control of burn wound infection. Clin Exp Immunol. 2009;156: $102-110$.

67. Zillmer R, Trostrup H, Karlsmark T, Ifversen P, Agren MS. Duration of wound fluid secretion from chronic venous leg ulcers is critical for interleukin-1alpha, interleukin-1beta, interleukin-8 levels and fibroblast activation. Arch Dermatol Res. 2011;303:601-606.

68. Brackman G, Coenye T. Quorum sensing inhibitors as anti-biofilm agents. Curr Pharm Des. 2015;21:5-11.

69. Ciofu O, Tolker-Nielsen T, Jensen PO, Wang H, Hoiby N. Antimicrobial resistance, respiratory tract infections and role of biofilms in lung infections in cystic fibrosis patients. Adv Drug Deliv Rev. 2015;85:7-23.

70. Davis SC, Ricotti C, Cazzaniga A, Welsh E, Eaglstein WH, Mertz PM. Microscopic and physiologic evidence for biofilm-associated wound colonization in vivo. Wound Repair Regen. 2008;16(1):23-29.

71. Thompson MG, Black CC, Pavlicek RL, et al. Validation of a novel murine wound model of Acinetobacter baumannii infection. Antimicrob Agents Chemother. 2014;58(3):1332-1342.

72. Calum H, Høiby N, Moser C. Burn mouse models. Psedomonas Methods and Protocols. 2014;1149:793-802.
Chronic Wound Care Management and Research

\section{Publish your work in this journal}

Chronic Wound Care Management and Research is an international, peer reviewed, open access, online journal publishing original research, reviews, editorials, and commentaries on the causes and management of chronic wounds and the major issues related to chronic wound management. Topics also include chronic wounds as comorbidities to other

\section{Dovepress}

conditions, patient adherence to therapy, and the economic burden of chronic wounds. The manuscript management system is completely online and includes a very quick and fair peer review system, which is all easy to use. Visit http://www.dovepress.com/testimonials.php to read real quotes from published authors. 